\title{
Multiscale dynamics of branching morphogenesis
}

\author{
Edouard Hannezo ${ }^{1}$ and Benjamin D. Simons $s^{2,3,4}$
}

1 Institute of Science and Technology Austria, Klosterneuburg, 3400, Austria

2 The Wellcome Trust/Cancer Research UK Gurdon Institute, University of Cambridge, Cambridge CB2 1QN, UK

3 Department of Applied Mathematics and Theoretical Physics, Centre for Mathematical Sciences, University of Cambridge, Wilberforce Road, Cambridge CB3 OWA, UK

4 The Wellcome Trust/Medical Research Council Stem Cell Institute, University of Cambridge, Cambridge CB2 1QN, UK

\section{Abstract}

Branching morphogenesis is a prototypical example of complex three-dimensional organ sculpting, required in multiple developmental settings to maximize the area of exchange surfaces. It requires, in particular, the coordinated growth of different cell types together with complex patterning to lead to robust macroscopic outputs. In recent years, novel multiscale quantitative biology approaches, together with biophysical modelling, have begun to shed new light of this topic. Here, we wish review some of these recent developments, highlighting the generic design principles that can be abstracted across different branched organs, as well as the implications for the broader fields of stem cell, developmental and systems biology.

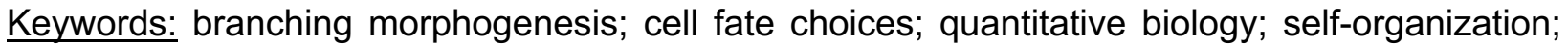
patterning instability

\section{$\underline{\text { Introduction }}$}

Beginning with Aristotle's comparison of veins and river drainage patterns, the process of branching morphogenesis, by which arborized networks are built during development, have fascinated experimentalists and theoreticians alike. Due to the seeming ubiquity of branched morphologies in epithelial tissues (including lung, pancreas, prostate, liver, kidney and mammary gland), in circulatory and lymphatic systems, and even at the level of individual cells (neurons, Drosophila tracheal cells and fungi), scientists have questioned the rules involved in the generation of these structures. From $\mathrm{Da}$ Vinci's area preserving rule for tree growth, to Mandelbrot's fractal lung ${ }^{1}$, these considerations remained a largely theoretical exercise, until advances of molecular biology, over the past 30 years, allowed for a much deeper characterization of the genetic interactions driving branching morphogenesis in vivo. However, how this information at the molecular level can be integrated across multiple length scales, to allow for the shaping and function of organs, is still unclear. The advent of organoid culture, or "organs-in-a-dish", has showed that several aspects of branching morphogenesis (from cell differentiation patterns to shape acquisition) can be recapitulated in minimal in vitro conditions ${ }^{2}$, underlining the need for a better understanding of such self-organization properties. This has started to be tackled at multiple different scales in parallel (Figure 1), with each specific question (what is the potency and behaviour of a single cell? what are the mechanisms of tip branching? how are large-scale properties of an organ determined?) calling for different theoretical (Table 1) and experimental approaches. Here, we aim to review our current understanding of branching morphogenesis, highlighting the generic design principles that can be abstracted at different scales (Figure 1) and across organs ${ }^{3-5}$. 
Although reconstructions of adult branched structures such as the vasculature and lung have been long known, a detailed and quantitative time course showing how such structures emerge during development has only appeared in the past decade, for instance in the lung, kidney, pancreas and mammary gland, thanks to the improvement of multi-scale imaging tools and reconstructions ${ }^{6,7}$. Combined with genetic perturbation assays, explant live-imaging and cellular lineage tracing methods, these studies have underlined the diversity of mechanisms generating branched structures at all scales. As it typically combines tightly patterned cellular fate choices, complex 3D shape changes and coordination between multiple tissue types to produce robust outcomes, branching morphogenesis thus emerges as an ideal playground to resolve some of the central questions confronting stem cell, developmental and systems biology.

\section{Table 1. Classes of models for branching morphogenesis}

Lineage-tracing models: Concentrating on the fate of individual marked cells and their progeny, these models seek to understand the patterns of cell fate choices (for instance between proliferation, differentiation or death) during development, and often engage genetic lineagetracing strategies. These approaches enable an estimation of the potency, dynamics and location of developmental precursors.

Reaction-diffusion models: Building on the work of Alan Turing, this class of mesoscopic models focusses not on cells, but on how cellular domains respond to the spatio-temporal properties of diffusing and reacting biochemical species. In specific parameter regimes of interactions and diffusion coefficients (and assuming that these are coupled to cellular growth), branched spatial patterns can emerge.

Mechanical models: Aimed at the same mesoscopic scale as reaction-diffusion systems, these type of models consider branching patterns as resulting from the mechanical properties of epithelial and mesenchymal tissues. Epithelial layers growing in a confined elastic environment are, for instance, predicted to spontaneously break symmetry and form folds at given length scales. Spatial patterns of epithelial tension/contractility and/or active migration forces have also been proposed to mediate branching events.

Network models: When the biological question is aimed at understanding what sets the largescale properties of a branching tree, it can be more convenient to model them as a network (independent of the underlying mechanical or reaction-diffusion properties of the trees). The network is then evolved with rules, which can be deterministic or stochastic. Such rules can be used to describe how a network grows (e.g. describing the branching and termination of tips), or remodels (e.g. based on fluid flow in a network).

\section{Cellular heterogeneity in branching morphogenesis}

At the cellular scale, a key question has been to understand the lineage relationships between the various cell types that generate branched organs. Combined with single-cell RNA sequencing, the advent of lineage tracing methods during the past decade have made it possible to physiologically assess the genealogy of fate choices made by individual cells: In mouse prostate, for instance, these studies have shown that the two ductal compartments that comprise the epithelium (the luminal and myoepithelial basal cells) consist of independently-maintained populations during homeostasis and regeneration ${ }^{8}$, while in the adult mouse lung alveoli, AT1 (alveolar type 1, squamous) cells are produced by specific populations of slow-cycling AT2 (alveolar type 2, surfactant producing) cells ${ }^{9}$. Such findings have important relevance for understanding regeneration ${ }^{10}$ and cancer initiation ${ }^{11}$, which typically occurs from a well-defined cell type. However, while lineage tracing is facilitated at homeostasis by the typically low rate of cell movements and turnover, performing it during branching morphogenesis has proved problematic, as the extensive morphogenetic movements entail clones typically being dispersed throughout the entire organ ${ }^{8}$. To meet these challenges, multiscale imaging methods have been 
developed in the last 5 years that allow for whole-organ reconstructions s, $^{72}$ together with single cell resolution to resolve fate markers ${ }^{9,13,14}$, combined with single-cell sequencing and statistical methods necessary to reconstruct clonal information despite fragmentation ${ }^{8,13,15}$.

During postnatal mammary gland development in mouse, these studies have revealed a surprising degree of sub-lineage segregation from early stages of branching morphogenesis, although rare events of cellular bipotency cannot be rigorously ruled out ${ }^{13,16-20}$. In the later stage of mouse pancreas development, branching morphogenesis is similarly mediated predominantly by pools of unipotent progenitors ${ }^{21}$. This raises an interesting conundrum: How do several distinct cell types cooperate within rapidly growing tip regions, through serial rounds of branching, without "overtaking" each other, whilst ensuring a robust and harmonious distribution of cell types within a patterned complex branched organ? During kidney morphogenesis, although the uteric bud consists of a single lineage of self-renewing progenitors, branching morphogenesis occurs via the highly coordinated expansion and cross-talk between epithelial cells and surrounding mesenchymal niches ${ }^{22}$ to give rise to both the collecting ductal structure and filtering nephrons. This cross-talk is underlined in lineage-ablation experiments, where the removal of a large fraction of mesenchymal nephron progenitors also decreased the branching rate of the epithelial ducts to allow for partial compensation ${ }^{23}$, a feature reproduced by mathematical models of epithelial/mesenchymal interactions ${ }^{24}$. Interestingly, the dynamics of nephron progenitor commitment was recently shown to be highly stochastic and reversible, combining extensive random cell migration to local niche signals ${ }^{25}$. It is tempting to speculate that bidirectional coupling between cell types, together with competition for limited niche access and stochastic reversibility, might be a generic feature of biological systems, endowing them robustness with respect to perturbation. This idea has only recently begun to be systematically investigated theoretically ${ }^{26,27}$, which is likely to yield generic insights into cell fate regulation. Moreover, tailoring our understanding of niche-epithelium interaction down to the single-cell level would help shed light on this. In the homeostatic renewal of mouse lung, it was shown recently that the stem cell potential of a small subset of AT2 cells is dictated by a niche signal from single Wnt-expressing fibroblasts ${ }^{28}$, calling for a more generic exploration in developmental settings ${ }^{14}$, which remains highly challenging.

More generally, the prevalence and role of molecular heterogeneity within branching tips has been underscored recently in multiple model systems. Epithelial dynamics in kidney displays extensive cell-to-cell heterogeneity of key branching factors such as $\operatorname{Ret}^{29}$. High Ret expression has been implicated, both in wild-type kidney and mosaic loss-of-function experiments, to prime cells to remain within tips upon the next branching event, with Ret-low cells left behind in ducts. However, Ret-high cells do not become enriched in tips over time, arguing that heterogeneity in Ret is actively maintained in the system ${ }^{29}$. In mammary morphogenesis, although terminal end buds consist of multiple populations of transcriptionally heterogeneous cells ${ }^{13,19}$ (some of which had been proposed to constitute a stem cell population), unbiased lineage tracing experiments and intravital live-imaging during pubertal growth are quantitatively consistent with a scenario in which each cell type in a tip behaves as single, equipotent population ${ }^{16}$. This does not preclude short-term biases in fate, which has been observed based on positional information and distance from the tip (although transcriptional signature could play a role as in kidney). However, it does argue that these must be actively resolved into long-term equipotency in the long-term. These findings echo strongly the homeostatic renewal strategy of intestinal crypts $^{30}$, in which fate of Lgr5+ cells are dictated on the short term by distance from the centre of the crypt, while functioning long-term as an equipotent population. In contrast to intestinal crypts, however, extensive cellular mixing has been observed in kidney (in both epithelium ${ }^{29}$ and mesenchyme ${ }^{25}$ ) and mammary gland morphogenesis ${ }^{16}$, which could help reconcile short- and long-term behaviour. Again, one can speculate that maintaining a large heterogeneous pool of progenitors could confer robustness to the system. Recent insights into the less-explored branching morphogenesis and patterning 
program at the cellular level of salivary ${ }^{31}$, thyroid ${ }^{32}$ and lacrimal ${ }^{33}$ glands are likely to help explore the potential generality of such principles.

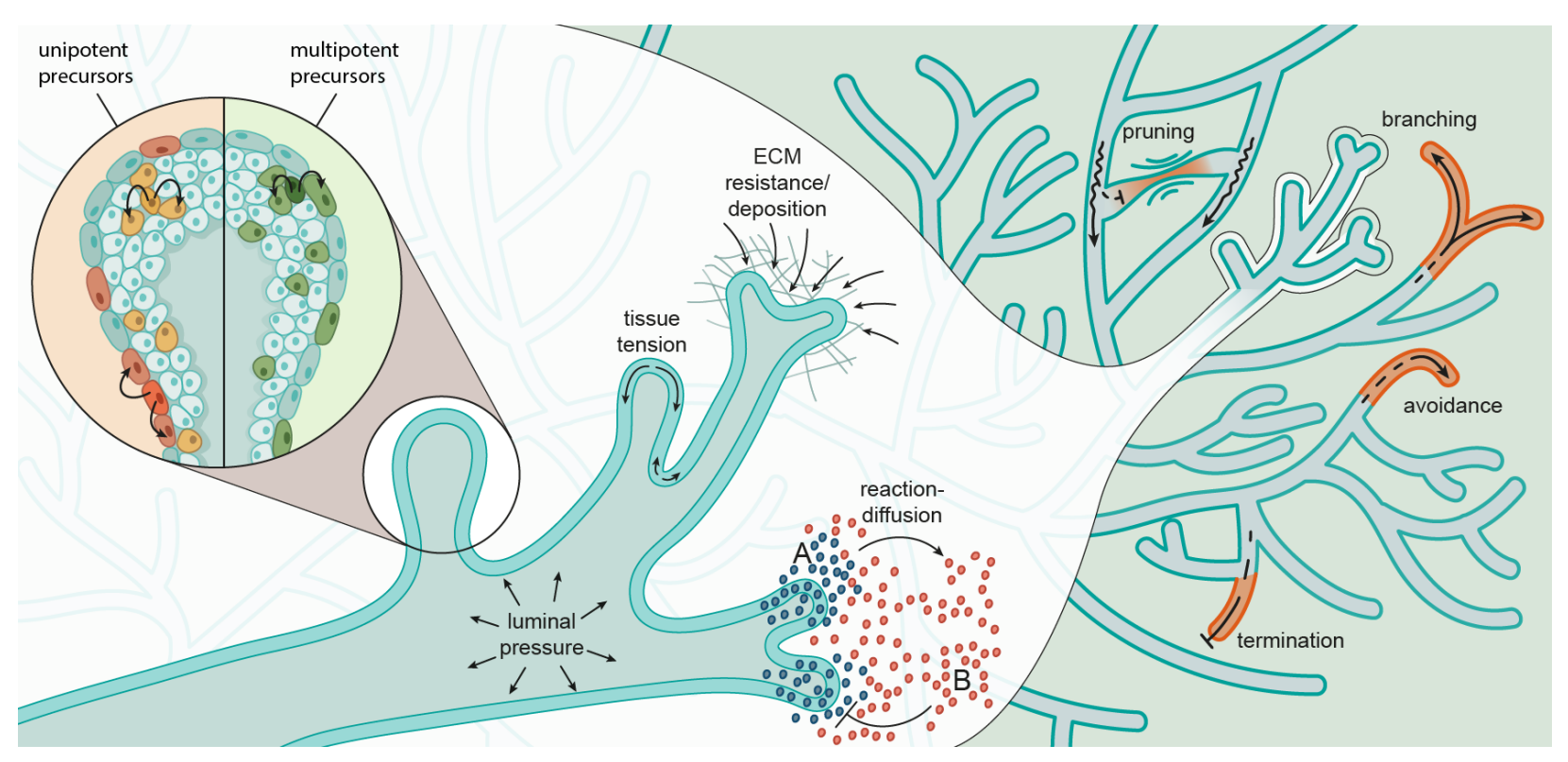

Fig. 1: Understanding the mechanisms of branching morphogenesis at multiple scales

Left-to-right: At the cellular level, branching morphogenesis proceeds from the collective and stochastic dynamics of heterogeneous progenitor compartments, whose potency can be assessed via lineage tracing experiments and statistical methods (left side: tracing of two distinct unipotent populations, right side: tracing of a single multipotent population).

At the mesoscopic level, the shape and growth of branching tips can be influenced by (i) the balance of forces between the growing tissue and its surroundings (such as the internal luminal pressure, external resistance and/or remodelling from the $\mathrm{ECM} /$ mesenchyme, active migratory forces from tip cells, or the internal stresses of the epithelium arising from patterns of cell proliferation and/or differential cytoskeletal tensions), or by (ii) the cross-reaction dynamics of diffusing morphogens (sketched by $A$ and $B$, produced resp. by the epithelial and mesenchymal cells) specifying patterns of cellular growth.

At the organ-level, the emergent large-scale properties of branching can be best understood via a coarse-grained model consisting of simple rules, such as network pruning based on minimization of fluid flow dissipation, stochastic or deterministic branching evens, and selfavoidance or self-termination of growing tips (each set of rules giving rise to markedly different network properties and more suited for a given organ).

\section{Mesoscopic mechanisms of branching morphogenesis}

At the mesoscopic scale, periodic rounds of tip elongation and branching have long been proposed to be mediated by reaction-diffusion type processes, which can reproduce well the deterministic branching patterns observed in early stage kidney and lung development ${ }^{34,35}$. These rely on Turing's classical theory of morphogenesis, with pairs of receptor/ligands (FGF10/FGFR2b in lung and GDNF/RET in kidney) with different diffusivity. Interestingly, this differential diffusivity was proposed ${ }^{35}$ in both cases to arise from the fact that the ligands Fgf10 and Gdnf are expressed (and diffuse) in mesenchymal tissues, while their receptors, Fgfrllb and Ret, are expressed in the epithelium (and largely immobile). Thus, although stated in molecular and "continuum" terms, 
such Turing models can therefore also be seen as a description of niche-epithelium interactions discussed at the cellular scale in the previous section. A strength of this type of receptor/ligand is its ability to explain why branching still proceeds when a uniform source of FGF10 is given to lung explants (despite its non-uniform distribution during in vivo branching), and has been shown to predict quantitatively the instantaneous growth pattern of kidney explants when simulating the model using the experimentally-segmented geometry at each given time ${ }^{35}$.

In recent years, a complementary and "mechanical" basis for branching morphogenesis has begun to emerge, to evaluate (both theoretically and experimentally) the role of mechanical forces in setting up the morphogenetic patterns ${ }^{36}$. In branching morphogenesis, these include differential apical-basal tensions in 3D vertex models ${ }^{37}$, E-cad mediated cell adhesion in multiple organs $^{38}$, Myosin II-independent cell migration in Drosophila trachea ${ }^{39}$ or luminal pressure forces in mouse lung ${ }^{40}$. The ECM has also emerged as an important regulator, both by providing external mechanical resistance during buckling instabilities ${ }^{41}$, as well as spatially-varying cues influencing epithelial dynamics via integrin signalling ${ }^{42,43}$. Interestingly, it was also shown that spatial modulation of tissue tension due to local patterns of cell differentiation played a key role in chick lung branching ${ }^{44}$, providing a potential link from biochemical and fate patterning to mechanical forces. Conversely, changes in tissue geometry originating from growth and tissue tension can impact on biochemical concentrations ${ }^{35,45}$. Thus, although the causality and respective importance of biochemical vs. mechanical signals in patterning has yet to be systematically and quantitatively investigated, understanding such mechano-chemical feedback loops is beginning to yield fascinating insights into branching morphogenesis. In lung morphogenesis, local forces have recently been involved in the patterning of the cell division axis during tubular morphogenesis ${ }^{46}$, while global pressure forces from inhalation movements have been shown to impact on alveolar differentiation into either type I (AT1) or II (AT2), with a subset of initially uncommitted cells exerting Fgf10-dependent protrusions that "protect" them from the fluidpressure induced stretching that characterizes future AT1 cells ${ }^{47}$.

\section{Large-scale design principles of branched networks}

Such mechano-chemical models typically concentrate on the symmetry-breaking properties of branching morphogenesis at the scale of single tips and branches. Although it is expected that these models are the right theory to describe branching morphogenesis at the mesoscopic scale, the sheer size of branched organs (consisting often at least of tens of thousands of tips and branches) can make such approaches intractable. A complementary view from statistical physics has been to adopt a coarse-grained approach, simplifying interaction rules at the mesoscopic scale to concentrate on the emergent properties of branching morphogenesis at the organ scale.

Although early lung and kidney development is highly deterministic, many other branched structures display little organism-to-organism stereotypy. The type of molecular and/or cellular "code" that could implement either architecture remain a key open question, a topic which has also attracted attention in neuroscience ${ }^{48}$. However, new classes of stochastic models have recently been proposed to study branching patterns in multiple systems ${ }^{49-51}$, and restrict the range of possible underlying biological mechanisms. These models describe branching morphogenesis as a stochastic process in which active tips explore space randomly in all directions while laying out ductal structures, branch, and are subject to local negative feedbacks on their growth, to avoid exponential uncontrolled expansion. Conceptually, the strength of such models is that they allows for a self-organizing tiling of space (albeit via highly inefficient space-filling) in a robust manner with respect to geometry, boundary conditions or global guiding cues; but also that it contains very few parameters to predict large-scale organ structure.

Although molecular implementation of such a design principle is bound to differ widely among branched organs, this model provides a potential unifying feature among seemingly 
disparate systems. In mouse mammary gland, large- and small-scale organ structure can be wellpredicted by a stochastic model where ducts provide termination signals on tip growth in a TGF $\beta$ dependent manner ${ }^{49}$. In pancreatic organoids, the early stages of branching have been proposed to rely on a similar tip-driven process with short-range inhibitory signal secreted by the epithelium $^{52}$, while clonal lineage tracing of late-stage embryonic branching morphogenesis are consistent with a branching and terminating model ${ }^{21}$ (although residual proliferative activity and side-branching persist within ducts). In kidney explants cultured in close proximity, growing tips terminate to avoid collision in a BMP7-dependent manner ${ }^{53}$, a crowding effect which has been shown to arise generically in aforementioned reaction-diffusion models ${ }^{35}$. Moreover, the morphogenesis of highly branched neurons, such as Purkinje cells ${ }^{50}$ in mouse and class IV sensory neurons in Drosophila ${ }^{51}$, have also been studied by proposing a similar paradigm, with random growth and branching of active tips, combined with tip/axon retraction upon physical contact with another branch (shown to be dependent on PKC in Purkinje cells ${ }^{50}$ ).

This raises the exciting hypothesis that these widely different classes of systems (single cells vs. multicellular organs, diffusive vs. physical inhibition) could belong to the same classes of models, or share "universal" features as defined in physics terms. However, additional investigations in other organs must still be performed to rigorously show this. In kidney morphogenesis, early and mid-embryonic stages have been shown to be highly regulated and stereotypic $^{6,54}$, while late-embryonic trees have been proposed to be non-deterministic ${ }^{55}$, and well fit by a stochastic model of branching and termination ${ }^{49}$; although further studies will be necessary to address the relative contribution of both during development.

In parallel, recent work on pancreas morphogenesis has suggested an alternative route to give rise to branched organs, where an initially connected central plexus remodels extensively to give rise to a tree-like morphology. In analogy with findings on angiogenesis ${ }^{56,57}$, this has been proposed to result from the minimization of viscous drag due to fluid flow within the structure ${ }^{58}$. As branched organs have a critical function in fluid transport, and given the aforementioned growing evidence for the role of fluid pressure in influencing tip branching ${ }^{40}$, it will be important in future studies to assess the interplay between growth processes and transport mechanisms, as well as achieve a better quantitative understanding of whether design principles in each system corresponds to a given optimality criterion ${ }^{56}$.

\section{Outlook:}

Recent advances in multiscale imaging and biophysical manipulations have made it possible to begin deciphering the dynamics of branching morphogenesis in vivo, from cellular dynamics to large-scale tissue architecture. Although the morphogenesis of different branched organs differs crucially in terms of underlying molecular circuits and the stochasticity vs. determinism of their mesoscopic rules, exciting similarities in design principles are emerging at different scales. These include the stochastic heterogeneity of behaviour and fate choices of their developmental precursors, reaction-diffusion frameworks driving symmetry breaking during branching, and self-organized large-scale patterning rules (Table 1). A key problem for future years will be to integrate this new knowledge into our understanding of the detailed molecular pathways driving branching morphogenesis. Given the recent progresses in organoid culture establishment $^{2,59}$, as well as the drastic improvements in clearing methods to image branching morphogenesis in human embryos ${ }^{60}$, one can anticipate a fruitful interaction between in vivo and in vitro systems to understand the emergence of complex organs during development, but also guide therapeutic applications in regenerative medicine and disease modelling.

The authors declare no conflict of interest. 
Funding information

Royal Society; Wellcome Trust, Grant/Award Number: 098357/Z/12/Z and 092096; CRUK, Grant/Award Number: C6946/ A14492

References and recommended reading:

Papers of particular interest published within the last two years have been highlighted as

** of outstanding interest

1. Weibel, E. R. Fractal geometry: a design principle for living organisms. Am. J. Physiol. 261, L361-L369 (1991).

2. Bredenoord, A. L., Clevers, H. \& Knoblich, J. A. Human tissues in a dish: The research and ethical implications of organoid technology. Science 355, (2017).

3. Varner, V. D. \& Nelson, C. M. Computational models of airway branching morphogenesis. Seminars in Cell and Developmental Biology 67, 170-176 (2017).

4. Lu, P. \& Werb, Z. Patterning mechanisms of branched organs. Science 322, 1506-1509 (2008).

5. Iber, D. \& Menshykau, D. The control of branching morphogenesis. Open Biol. 3, 130088 (2013).

6. Short, K. M. et al. Global quantification of tissue dynamics in the developing mouse kidney. Dev. Cell 29, 188-202 (2014).

7. Metzger, R. J., Klein, O. D., Martin, G. R. \& Krasnow, M. A. The branching programme of mouse lung development. Nature 453, 745-750 (2008).

8. Wuidart, A. et al. Quantitative lineage tracing strategies to resolve multipotency in tissuespecific stem cells. Genes Dev. 30, 1261-1277 (2016).

9. Desai, T. J., Brownfield, D. G. \& Krasnow, M. A. Alveolar progenitor and stem cells in lung development, renewal and cancer. Nature 507, 190-194 (2014).

10. Wang, Y. et al. Pulmonary alveolar type I cell population consists of two distinct subtypes that differ in cell fate. Proc. Natl. Acad. Sci. 115, 2407-2412 (2018).

11. Beck, B. \& Blanpain, C. Unravelling cancer stem cell potential. Nature Reviews Cancer 13, 727-738 (2013).

12. Short, K., Hodson, M. \& Smyth, I. Spatial mapping and quantification of developmental branching morphogenesis. Development 140, 471-478 (2013).

13. Scheele, C. L. G. J. et al. Identity and dynamics of mammary stem cells during branching morphogenesis. Nature 542, 313-317 (2017).

**This paper combines clonal lineage tracing, single-cell RNA sequencing and whole-organ reconstruction to define the multiscale stem cell dynamics during the postnatal development of the mouse mammary gland epithelium.

14. Kumar, M. E. et al. Defining a mesenchymal progenitor niche at single-cell resolution. Science (80-. ). 346, (2014).

15. Rulands, S. et al. Universality of clone dynamics during tissue development Supplementary Material. Nat. Phys. 1-6 (2018). doi:10.1038/s41567-018-0055-6

16. Rios, A. C., Fu, N. Y., Lindeman, G. J. \& Visvader, J. E. In situ identification of bipotent stem cells in the mammary gland. Nature 506, 322-327 (2014).

17. Van Keymeulen, A. et al. Distinct stem cells contribute to mammary gland development and maintenance. Nature 479, 189-193 (2011).

18. Lilja, A. M. et al. Clonal analysis of Notch1-expressing cells reveals the existence of unipotent stem cells that retain long-term plasticity in the embryonic mammary gland. Nat. Cell Biol. 20, 677-687 (2018).

19. Davis, F. M. et al. Single-cell lineage tracing in the mammary gland reveals stochastic 
clonal dispersion of stem/progenitor cell progeny. Nat. Commun. 7, 13053 (2016).

20. Wuidart, A. et al. Early lineage segregation of multipotent embryonic mammary gland progenitors. Nat. Cell Biol. 20, 666-676 (2018).

21. Sznurkowska, M. K. et al. Defining Lineage Potential and Fate Behavior of Precursors during Pancreas Development. Dev. Cell (2018). doi:10.1016/j.devcel.2018.06.028

22. Short, K. M. \& Smyth, I. M. The contribution of branching morphogenesis to kidney development and disease. Nat. Publ. Gr. 12, 754-767 (2016).

23. Cebrian, C., Asai, N., D'Agati, V. \& Costantini, F. The number of fetal nephron progenitor cells limits ureteric branching and adult nephron endowment. Cell Rep. 7, 127-137 (2014).

24. Zubkov, V. S. et al. A spatially-averaged mathematical model of kidney branching morphogenesis. J. Theor. Biol. 379, 24-37 (2015).

25. Lawlor, K. T. et al. Nephron progenitor commitment is a stochastic process influenced by cell migration. Elife 8, (2019).

** This study explores the dynamics of nephron progenitor commitment during kidney morphogenesis showing plasticity due to stochastic movements in and out of a niche.

26. Zhou, X. et al. Circuit Design Features of a Stable Two-Cell System. Cell 172, 744757.e17 (2018).

27. Jörg, D., Kitadate, Y., Yoshida, S., arXiv, B. S. preprint \& 2019, undefined. Competition for Stem Cell Fate Determinants as a Mechanism for Tissue Homeostasis. arxiv.org

28. Nabhan, A. N., Brownfield, D. G., Harbury, P. B., Krasnow, M. A. \& Desai, T. J. Singlecell Wnt signaling niches maintain stemness of alveolar type 2 cells. Science (80-. ). 359, 1118-1123 (2018).

29. Riccio, P., Cebrian, C., Zong, H., Hippenmeyer, S. \& Costantini, F. Ret and Etv4 Promote Directed Movements of Progenitor Cells during Renal Branching Morphogenesis. PLoS Biol. 14, e1002382 (2016).

30. Ritsma, L. et al. Intestinal crypt homeostasis revealed at single-stem-cell level by in vivo live imaging. Nature 507, 362-365 (2014).

31. Chatzeli, L., Gaete, M. \& Tucker, A. S. Fgf10 and Sox9 are essential for the establishment of distal progenitor cells during mouse salivary gland development. Development 144, 2294-2305 (2017).

32. Liang, S. et al. A branching morphogenesis program governs embryonic growth of the thyroid gland. Development 145, dev146829 (2018).

33. Farmer, D. T. et al. Defining epithelial cell dynamics and lineage relationships in the developing lacrimal gland. Development 144, 2517-2528 (2017).

34. Xu, H., Sun, M. \& Zhao, X. Turing mechanism underlying a branching model for lung morphogenesis. PLoS One 12, e0174946 (2017).

35. Menshykau, D. et al. Image-based modeling of kidney branching morphogenesis reveals GDNF-RET based Turing-type mechanism and pattern-modulating WNT11 feedback. Nat. Commun. 10, 239 (2019).

** This paper combines in vivo and in vitro experiments with modelling to show that the patterns of early kidney branching can be recapitulated quantitatively by a Turing mechanism.

36. Shyer, A. E. et al. Villification: How the gut gets its villi. Science (80-. ). 342, 212-218 (2013).

37. Okuda, S., Miura, T., Inoue, Y., Adachi, T. \& Eiraku, M. Combining Turing and 3D vertex models reproduces autonomous multicellular morphogenesis with undulation, tubulation, 
and branching. Sci. Rep. 8, 2386 (2018).

38. Daley, W. P. et al. Btbd7 is essential for region-specific epithelial cell dynamics and branching morphogenesis in vivo. Development 144, 2200-2211 (2017).

39. Ochoa-Espinosa, A., Harmansa, S., Caussinus, E. \& Affolter, M. Myosin II activity is not required for Drosophila tracheal branching. Development 144, 2961-2968 (2017).

40. Nelson, C. M. et al. Microfluidic chest cavities reveal that transmural pressure controls the rate of lung development. Development 144, 4328-4335 (2017).

** This paper develops bioengineered microfluidic cavities to show how luminal pressure can coordinate lung morphogenesis by setting globally the rate and timing of branching events.

41. Varner, V. D., Gleghorn, J. P., Miller, E., Radisky, D. C. \& Nelson, C. M. Mechanically patterning the embryonic airway epithelium. Proc. Natl. Acad. Sci. 112, 9230-9235 (2015).

42. Shih, H. P. et al. Report ECM Signaling Regulates Collective Cellular Dynamics to Control Pancreas Branching Report ECM Signaling Regulates Collective Cellular Dynamics to Control Pancreas Branching Morphogenesis. CellReports 14, 169-179 (2016).

43. Hsu, J. C. et al. Region-specific epithelial cell dynamics during branching morphogenesis. Dev. Dyn. 242, 1066-1077 (2013).

44. Kim, H. Y. et al. Localized Smooth Muscle Differentiation Is Essential for Epithelial Bifurcation during Branching Morphogenesis of the Mammalian Lung Short Article Localized Smooth Muscle Differentiation Is Essential for Epithelial Bifurcation during Branching Morphogenes. Dev. Cell 34, 719-726 (2015).

45. Shyer, A. E. et al. Bending Gradients : How the Intestinal Stem Cell Article Bending Gradients : How the Intestinal Stem Cell Gets Its Home. Cell 161, 569-580 (2015).

46. Tang, Z. et al. Mechanical Forces Program the Orientation of Cell Division during Airway Tube Morphogenesis. Developmental Cell 44, 313-325.e5 (2018).

47. $\mathrm{Li}, \mathrm{J}$. et al. The Strength of Mechanical Forces Determines the Differentiation of Alveolar Epithelial Cells. Dev. Cell 44, 297-312.e5 (2018).

** This paper provides a link between the differentiation of alveolar cells during lung morphogenesis and their mechanical properties.

48. Hassan, B. A. \& Hiesinger, P. R. Beyond Molecular Codes: Simple Rules to Wire Complex Brains. Cell 163, 285-291 (2015).

49. Hannezo, E. et al. A Unifying Theory of Branching Morphogenesis. Cell 171, $242-$ 255.e27 (2017).

**This study proposes that the statistical organization of several branched epithelial tissues can be defined quantitatively by simple self-organizing principles based on local rules.

50. Fujishima, K., Horie, R., Mochizuki, A. \& Kengaku, M. Principles of branch dynamics governing shape characteristics of cerebellar Purkinje cell dendrites. Development 139, 3442-3455 (2012).

51. Ganguly, S., Trottier, O., Liang, X., Bowne-Anderson, H. \& Howard, J. Morphology of Fly Larval Class IV Dendrites Accords with a Random Branching and Contact Based Branch Deletion Model. arxiv.org (2016).

52. Dahl-Jensen, S. B., Figueiredo-Larsen, M., Grapin-Botton, A. \& Sneppen, K. Short-range 
growth inhibitory signals from the epithelium can drive non-stereotypic branching in the pancreas. Phys. Biol. 13, 16007 (2016).

53. Davies, J. A., Hohenstein, P., Chang, C. H. \& Berry, R. A self-avoidance mechanism in patterning of the urinary collecting duct tree. BMC Dev. Biol. 14, 1-12 (2014).

54. Lefevre, J. G. et al. Branching morphogenesis in the developing kidney is governed by rules that pattern the ureteric tree. dev. biologists.org 4377-4385 (2017).

doi:10.1242/dev.153874

55. Sampogna, R. V., Schneider, L. \& Al-Awqati, Q. Developmental Programming of Branching Morphogenesis in the Kidney. J. Am. Soc. Nephrol. 26, 2414-2422 (2015).

56. Tekin, E., Hunt, D., Newberry, M. G. \& Savage, V. M. Do Vascular Networks Branch Optimally or Randomly across Spatial Scales? PLoS Comput. Biol. 12, 1-28 (2016).

57. Chen, Q. et al. Haemodynamics-Driven Developmental Pruning of Brain Vasculature in Zebrafish. PLoS Biol. 10, (2012).

58. Dahl-Jensen, S. B. et al. Deconstructing the principles of ductal network formation in the pancreas. PLoS Biol. 16, e2002842 (2018).

59. Chen, Y. W. et al. A three-dimensional model of human lung development and disease from pluripotent stem cells. Nat. Cell Biol. 19, 542-549 (2017).

60. Belle, M. et al. Tridimensional Visualization and Analysis of Early Human Development. Cell 169, 161-173.e12 (2017).

** This work uses clearing methods to provide a high-resolution, multiscale atlas of human embryo morphogenesis, and in particular the branching of nerves, lung and kidney. 